\title{
Antiangiogenic response after $70 \%$ hepatectomy and its relationship with hepatic regeneration and angiogenesis in rats
}

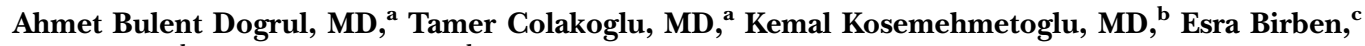
Elif Yaman, ${ }^{\mathbf{d}}$ Gokhan Gedikoglu, ${ }^{\mathbf{b}}$ and Osman Abbasoglu, MD, ${ }^{\mathbf{a}}$ Ankara, Turkey

Background. The aim of this study was to evaluate the antiangiogenic response and its relation to regeneration and angiogenesis after $70 \%$ hepatectomy in a rat model.

Methods. Sixty-four Wistar albino rats were included in the study. Animals were allocated into 8 groups $(\mathrm{n}=8)$. After a $70 \%$ hepatectomy, liver regeneration, angiogenesis, and antiangiogenic response were evaluated in the remnant liver on days $0,1,2,3,5,7,10$, and 14. Regeneration and angiogenesis were determined with immunoreactivity to proliferating cell nuclear antigen and vascular endothelial growth factor. Antiangiogenic response was evaluated by detecting collagen $18 \mathrm{~m} R \mathrm{RA}$ with reverse transcriptase polymerase chain reaction.

Results. We showed that liver regeneration peaked at day 1, whereas angiogenesis in the periportal and perisinusoidal areas reached their peak values on days 3 and 7 , respectively. Both regeneration and angiogenic activity around perisinusoidal hepatocytes returned to basal activity on the day 10.

Antiangiogenic response first appeared on day 5, reached a peak on day 10, and returned to basal values on day 14.

Conclusion. Collagen 18 mRNA expression is present in the normal liver during the regenerative process. We suggest that the stimulus that causes the cessation of regeneration process may come from hepatocytes, and collagen 18 produced by hepatocytes may modulate this event by inhibiting the angiogenesis. (Surgery 2010;147:288-94.)
\end{abstract}

From the Departments of General Surgery ${ }^{a}$ and Pathology, ${ }^{b}$ and Pediatric Allergy and Asthma Research Laboratory, ${ }^{c}$ Medical School, Hacettepe University, Ankara; Department of Molecular Biology and Genetics, Bilkent University, ${ }^{d}$ Ankara, Turkey

THE LIVER is unique in restoration of its anatomic volume and mass after toxic injury or surgical resection. Liver responds to these changes by regeneration. ${ }^{1}$ The optimal mass that the liver has to reach after partial hepatectomy or transplantation depends on predetermined parameters such as liver/body mass ratio. ${ }^{2-4}$ Time to restore liver mass depends on liver function rather than anatomy. $1,5-8$

There is a close interaction between liver regeneration and angiogenesis. After partial

Financial support of this study was supplied by Hacettepe University Scientific Research Unit (06 D02 101 004).

Accepted for publication October 5, 2009.

Reprint requests: Ahmet Bulent Dogrul, MD, Hacetepe Universitesi Tıp Fakültesi, Genel Cerrahi AD 06100, S1hhiye/Ankara, Turkey. E-mail: ahmetdogrul@yahoo.com.

0039-6060/\$ - see front matter

(c) 2010 Mosby, Inc. All rights reserved.

doi:10.1016/j.surg.2009.10.015 hepatectomy, first hepatocyte regeneration and then angiogenesis occur, and the liver reaches its optimal functional volume and mass between postoperative days 7 and 10 in rats. ${ }^{2,9-11}$ Interestingly, liver regeneration does not cause pathologic angiogenesis or growth out of control. When the liver reaches its optimal functional size, regeneration stops. ${ }^{10}$

In 1997, O'Reilly et al identified endostatin, which is an endogenous angiogenesis inhibitor produced by hemangioendothelioma. Endostatin was shown to be identical to the carboxy terminal of collagen 18. Systemic administration of endostatin inhibits potently angiogenesis and tumor growth and maintains metastases at a microscopic size. ${ }^{12}$

The liver was shown to be the main site of collagen 18 synthesis, and hepatocytes in the normal liver and satellite cells in the cirrhotic liver have been reported to be the important sources of this type of collagen. ${ }^{13}$ Collagen 18 is the only type of collagen known to be synthesized by hepatocytes. ${ }^{14}$ 
Although antiangiogenetic activity of neoplasms and antiangiogenic therapy for many types of solid neoplasms have been well studied after the discovery of endogenous angiogenesis inhibitors, to the best of our knowledge, no study in the literature has demonstrated antiangiogenic activity of the liver during normal liver regeneration. Most of the studies have focused on dependence of liver regeneration on angiogenesis. ${ }^{10,15-17}$

The aim of this study was to evaluate the antiangiogenic activity of the liver and its relation to regeneration and angiogenesis after $70 \%$ hepatectomy in rats.

\section{MATERIALS AND METHODS}

A total of 64 adult female Wistar albino rats weighing 250-350 g used in the study were fed with standard diet and water ad libitum and kept under standard room temperature at $22-24^{\circ} \mathrm{C}$ and in a 12-hr light/dark cycle. Animals were fasted for $8 \mathrm{hr}$ before hepatectomy. The Institutional Committee of Ethics of Animal Experiments at Hacettepe University approved the study in accordance of Declaration of Helsinki and institutional guide for use of laboratory animals (2005/72-5).

Experimental design. Sixty-four female rats were divided randomly into 8 groups of 8 rats each. Seventy percent hepatectomy was performed under $5 \mathrm{mg} / \mathrm{kg}$ xylazine (Rompun Bayer, Turkey) and $30 \mathrm{mg} / \mathrm{kg}$ ketamine hydrocloride (Ketalar Panker Davis, Turkey) anesthesia according to the method described by Higgins and Anderson. ${ }^{18}$ Groups were numbered according to day that the rats were sacrificed. After the $70 \%$ hepatectomy animals were sacrificed on days $0,1,2,3,5,7,10$, and 14 respectively. All groups were operated on during the same period during the light-dark cycle. Regenerating remnant livers were removed and specimens were fixed in $10 \%$ formalin solution for immunohistochemistry; a portion of liver was kept at $-80^{\circ} \mathrm{C}$ for reverse transcriptase polymerase chain reaction examination (RT-PCR).

Regenerative and angiogenetic activity of the remnant liver was evaluated by immunohistochemistry for proliferating cell nuclear antigen (PCNA) and vascular endothelial growth factor (VEGF). Regenerating livers were fixed in $10 \%$ formalin overnight and then embedded in paraffin blocks. Sections from the widest surface of the liver, each 4 $\mu \mathrm{m}$ thick, were cut. Antiangiogenetic activity was evaluated by RT-PCR technique for collagen 18; no good antibody was available for Western blot, Eliza, or immunohistochemistry for collagen 18 protein.

PCNA immunohistochemistry. PCNA (NeoMarkers; Cat. \#MS-106-P; LabVision Corp., Fremont,
CA) staining was performed on formalin-fixed, paraffin-embedded, $4-\mu \mathrm{m}$ thick sections using standard staining procedures. After deparaffinization and antigen retrieval with boiling in citrate buffer $(\mathrm{pH} 6)$, the sections were incubated with primary antibody according to the manufacturer's instructions and then processed by biotinylated goat anti-mouse antibodies followed by streptavidin conjugated to horseradish peroxidase (Polyvalent UltraTek Lab Pack Kit; ScyTek Laboratories Inc., Logan, UT) with the use of diaminobenzidine as the chromogen (DakoCytomation, Glostrup, Denmark). Nuclei stained in brown were considered as positive for PCNA. The PCNA labeling index was calculated as proportion of PCNA-positive hepatocytes per 1000 hepatocytes counted under a light microscope at $40 \times$ magnification.

VEGF immunohistochemistry. Immunohistochemical staining for VEGF on formalin-fixed and paraffin-embedded, $4-\mu \mathrm{m}$ thick, sections, was performed with anti-VEGF antibody (NeoMarkers VEGF Ab-1, Cat. \#RB-222-R7). After deparaffinization and antigen retrieval with boiling in citrate buffer ( $\mathrm{pH} \mathrm{6)}$, the sections were incubated with primary antibody according to the manufacturer's instructions and then processed by biotinylated goat anti-mouse antibodies followed by streptavidin conjugated to horseradish peroxidase (Polyvalent UltraTek Lab Pack Kit; ScyTek Laboratories) with the use of diaminobenzidine as the chromogen (DakoCytomation). Slides were examined by two blinded pathologists. VEGF staining was encountered mainly in periportal and perisinusoidal areas, and a semiquantitative analysis for VEGF staining was performed according to the degree of VEGF positive cell layer around these areas. Accordingly, VEGF staining in periportal areas was classified into 4 groups: 0 , no staining; $1+$, only one layer of VEGF positive hepatocytes around portal triad; 2+, 2 cell layer thickness of VEGF positive cells; and 3+, equal to or more than 3 layers of hepatocytes positive for VEGF around portal areas. Perisinusoidal staining of VEGF was assessed as follows: 0 , no staining; 1+, VEGF positivity in $<25 \%$ of perisinusoidal hepatocytes; $2+$, VEGF positivity in $25-50 \%$ of perisinusoidal hepatocytes; $3+$, VEGF expression in majortity of perisinusoidal hepatocytes, including pericentral areas.

Determination of anti-angiogenetic activity. Collagen 18 messenger ribonucleic acid (mRNA) was analyzed with RT-PCR to determine the anti-angiogenic activity in the regenerating liver after $70 \%$ hepatectomy. Total RNA was extracted from tissue samples with RNeasy mini kit (RNAaz, QIAGEN, Valencia, CA) according to the instructions of the 
Table. Sequence of primers used in PCR reactions and product sizes and annealing temperatures

\begin{tabular}{ccc}
\hline Primary sequences & PCR PRODUCT size $($ bp $)$ & Annealing temperature $\left({ }^{\circ}\right.$ C $)$ \\
\hline Rat B-actin F 5'-TTGTAACCAACTGGGACGATATGG-3' & 764 \\
$\quad$ Rat B-actin R 5'-GATCTTGATCTTCATGGTGCTAGG-3' & 58 \\
Rat GAPDH F 5'-AATGCATCCTGCACCACCAA-3' & 500 & 56 \\
Rat GAPDH R 5'-GTAGCCATATTCATTGTCATA-3' & \\
Rat Col18 F 5'-GGCGAGCAGATGACATCTT-3' & 413 \\
$\quad$ Rat Col18 R 5'-CGCCGGAGAACAGGGTGT-3' & & 58 \\
\hline
\end{tabular}

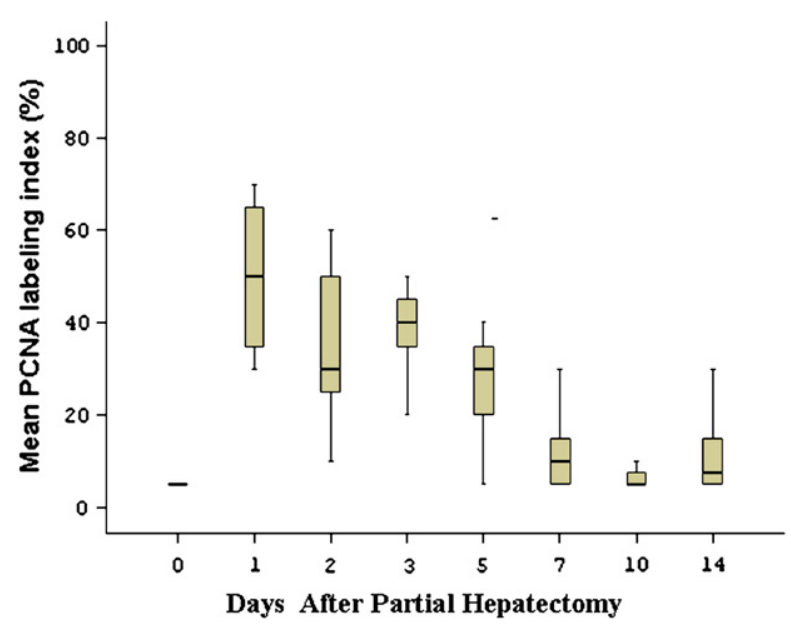

Fig 1. PCNA labeling index of hepatocytes reached its peak value on the first day. It remained high for 7 days compared to its basal value and returned to its basal value on day 10 .

manufacturer. Tissue samples were homogenized with pestle in RNA liquid nitrogen and lysed by using RNA lysis buffer. Homogenized lysate was loaded onto RNeasy spin column and centrifuged. After washing steps, total RNA was dissolved in RNAse free water. RNA amounts were measured at 260 and $280 \mathrm{~nm}$ in UV spectrophotometer. Total RNA (1000 ng) was reverse-transcribed at $42^{\circ} \mathrm{C}$ for $1 \mathrm{hr}$ using ImProm-II ${ }^{\mathrm{TM}}$ Reverse Transcription System from Promega (Madison, WI). Polymerase chain reaction reactions with rat $\beta$-actin primers and glyceraldehyde 3-phospho dehydrogenase (GAPDH) were used to confirm the presence of equal amount of cDNA for each reactions. After equalization of samples using the housekeeping genes $\beta$-actin and GAPDH, collagen 18 gene was amplified with Taq polymerase (Promega) at final concentration of $1.5 \mathrm{mM} \mathrm{MgCl} 2$. Sequence of primers used in PCR reactions, product sizes, and annealing temperatures are listed in the Table. PCR fragments were then subjected to electrophoresis on a $1.5 \%$ agarose gel containing ethidium bromide, visualized by ultraviolet transillumination, and captured by imaging system. The blots were obtained in all rats.
Quantification of RT-PCR results. Quantification of RT-PCR results was performed by the Bio-Profil V99 (Chemi-Capt 2000 Version 12.8, Bio-Profil Bio-1D Version 11.9; Vilber Lourmat, Marne la Vallée, France) analyzer program. Background activity on the ultraviolet images were ignored. Color density of the bands were calculated by the program and the ratio of C18 band color density to corresponding $\beta$-actin or GAPDH color density was calculated.

Statistical analysis. The comparisons of statistical significance among groups were performed with the Kruskal-Wallis and the Mann-Whitney $U$ test between independent groups. The day on which $P<.05$ for the first time was considered as a significant increase and the day on which $P>.1$ was considered as returning to basal activity. Continuous variables are presented as median values with interquartile ranges (boxes) and ranges.

\section{RESULTS}

Liver regeneration after $\mathbf{7 0 \%}$ hepatectomy. PCNA labeling index was less than $5 \%$ in hepatocytes on day 0 . A dramatic increase in the PCNA labeling index of hepatocytes were observed on day 1 which was its peak value $(P=.003)$. This index gradually decreased but remained significantly greater than basal values until day 7 . On day 10 , the PCNA labeling index of hepatocytes reached its basal value and remained low until day 14 $(P=.143$; Figs 1 and 2).

Angiogenic activity of the remnant liver. Angiogenic activity in periportal hepatocytes: There were very few VEGF-positive hepatocytes on day 1 . The increased expession of VEGF on day 0 was attributed to operative trauma caused by the hepatectomy. When compared with day 1, expression of VEGF on periportal hepatocytes peaked on day 3 $(P=.001)$ and decreased to the basal value on day 5 and day 7 was not significant when compared with day 1 (Figs 3 and 4).

Angiogenic activity in perisinusoidal hepatocytes: There were very few VEGF-positive hepatocytes on day 1. The increased expession of VEGF on day 0 was attributed to operative trauma caused by 

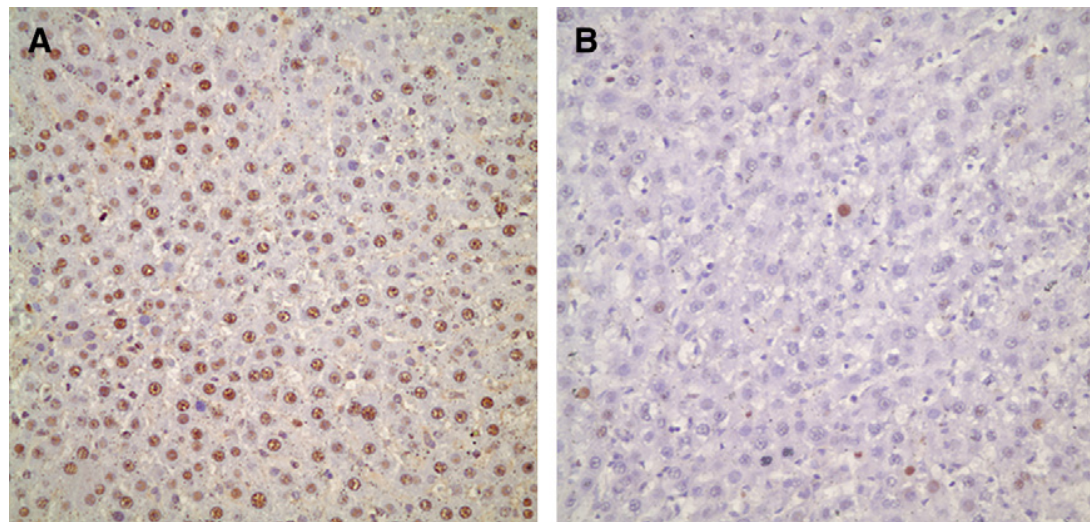

Fig 2. (A) Immunohistochemical staining for PCNA . On the first day after $70 \%$ hepatectomy more than $90 \%$ of hepatocytes were positive for PCNA. (B) On the tenth day less than $5 \%$ of hepatocytes were positive for PCNA.

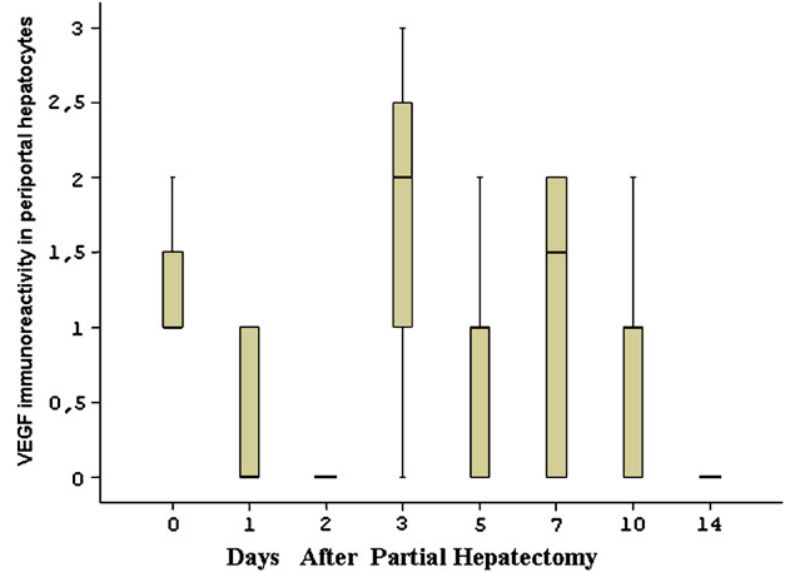

Fig 3. VEGF immunoreactivity in periportal hepatocytes reached its peak value on day 3 and returned to its basal value on day 5 .

hepatectomy. Angiogenesis around the perisinusoidal hepatocytes began on day 3 , gradually increased and reached its peak value on day 7 $(P=.039)$ and returned to its basal value on day 10 compared to day 1 . Changes on day 3 and 5 were not statistically significant (Fig 4 and 5).

Antiangiogenic activity of the remnant liver after 70\% hepatectomy. Anti-angiogenic activity of the liver after $70 \%$ hepatectomy was first detected on day 3. The first significant increase occurred on day $5(P=.011)$ and reached its peak value on day $10(P=.001)$ and returned to basal value on day 14 $(P=.34$; Figs 6 and 7$)$.

\section{DISCUSSION}

In this study, we showed that antiangiogenic activity in the remnant liver was seen initially on day 3 , and a significant increase was noted on day 5 after $70 \%$ hepatectomy. On day 5 , the angiogenic activity declined around periportal hepatocytes and started to increase around the perisinusoidal hepatocytes. In contrast, peak antiangiogenic activity was detected on day 10 , corresponding to the return of angiogenic activity to basal values around perisinusoidal hepatocytes and the day on which the liver reached its optimal functional volume (day 7-10)., ${ }^{1,2}$

Previously, it has been shown that when angiogenic agents are administered, liver regeneration increases by $16-42 \% .{ }^{10}$ In contrast, when antiangiogenic agents are administered, liver regeneration decreases by $46-74 \% .{ }^{10,17}$ These findings support the idea of dependence of liver regeneration on angiogenesis. While angiogenic agents increase liver regeneration in the early days of liver regeneration ( $42 \%$ on day 2 and $16 \%$ on day 8 ), the most pronounced effects of antiangiogenic agents on liver regeneration appear in the later days of regeneration (decreases regeneration on days 2 and 8 by $46 \%$ and $64 \%$, respectively, and $50 \%$ on day 7$).{ }^{10,17}$ These late, negative effects of antiangiogenic agents on regeneration may be explained by appearance of angiogenesis later than regeneration after partial hepatectomy. However, endogenous antiangiogenic activity during normal liver regeneration after partial hepatectomy has not been shown.

Peak values of regeneration and angiogenesis were detected on days 1 and 3 , respectively, in this study. There was a 48 -hr delay between regeneration and angiogenesis that can be explained by independence of regeneration from angiogenesis at an early time of regeneration. At a later stage, regeneration may become dependent on angiogenesis. Angiogenesis is stimulated by angiogenic factors such as VEGF and fibroblast growth factor (FGF) which are expressed by regenerating hepatocytes. 

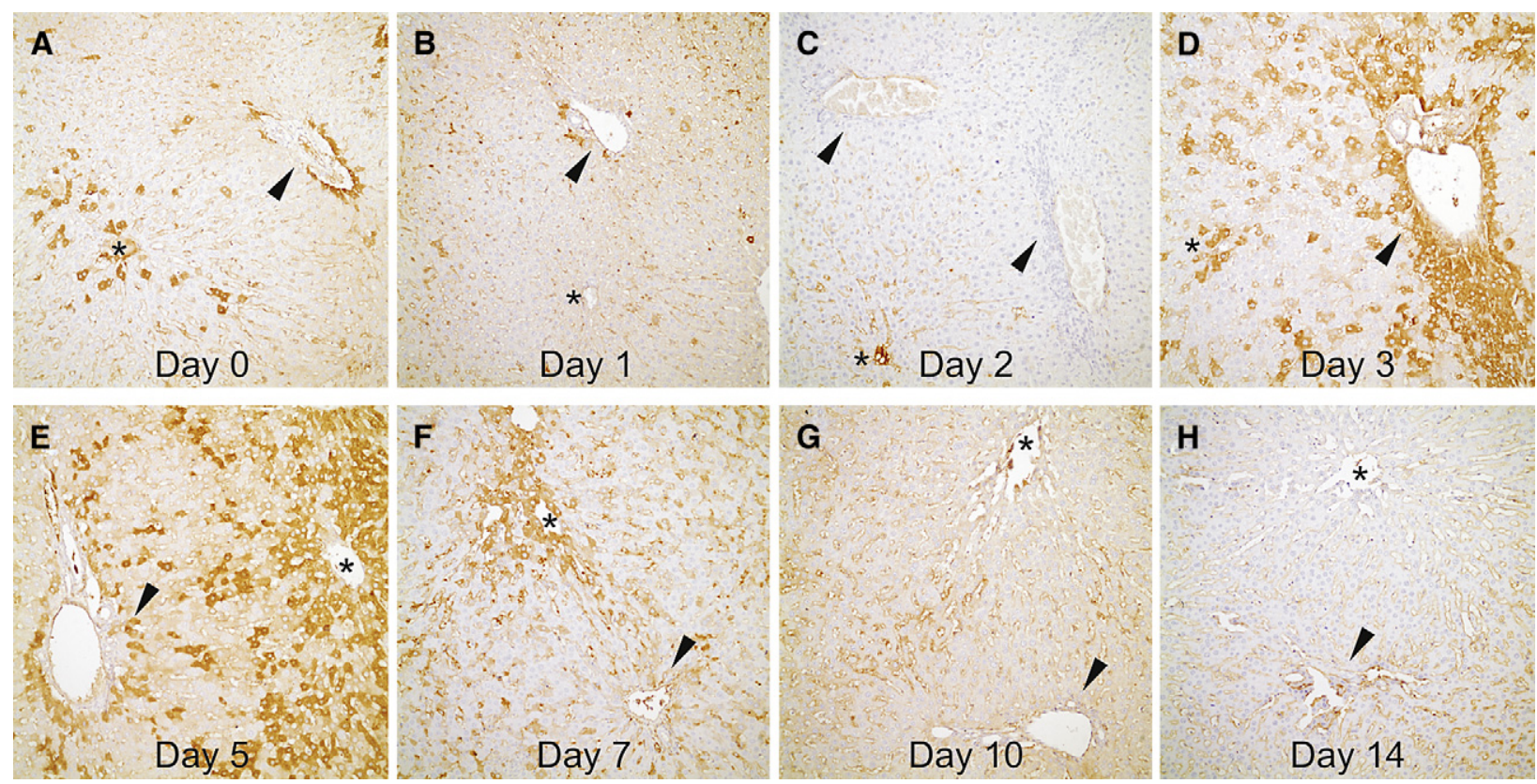

Fig 4. Representative sections of rat livers showing VEGF distribution (arrowheads, portal areas; asterisks, central veins) within day 0 and 14 (all figures; $200 \times$ ): $(A) 1+$ VEGF expression in both periportal and pericentral/sinusoidal areas. $(B, C)$ Second and third days with minimal VEGF activity. $(D)$ 2-3 layers of VEGF positive hepatocytes (3+) around portal vein, comparing to $1+$ expression in pericentral and sinusoidal areas. $(E)$ transposition of VEGF activity from periportal areas $(1+)$ to pericentral areas $(3+)$ on day 5. (F) VEGF activity in periportal areas ceased. Note the continuing activity around central vein. $(G, H)$ VEGF expression diminished in rat liver after day 10 of partial hepatectomy.

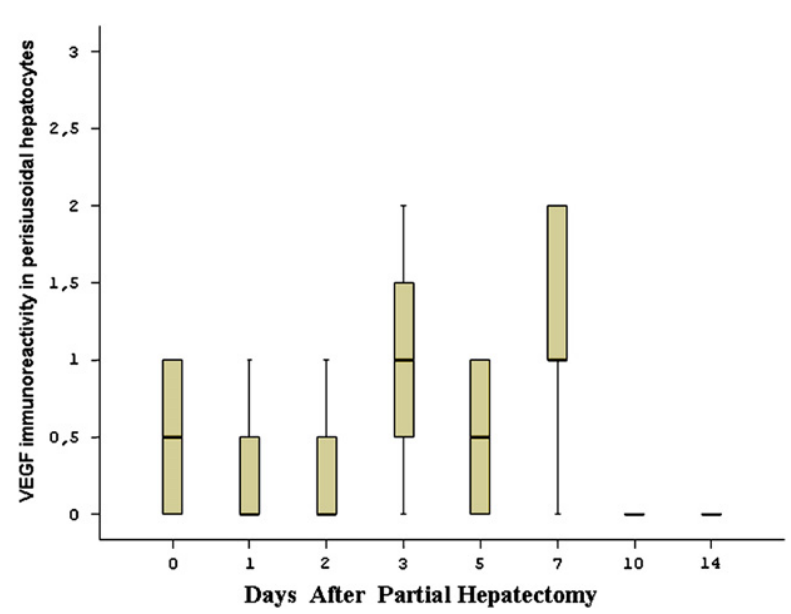

Fig 5. VEGF immunoreactivity in perisinusoidal hepatocytes increased on day 3 but reached peak value on day 7 and returned to basal value on day 10 .

This 48-hr difference between the peak value of angiogenesis and regeneration has been also shown in other models of $70 \%$ hepatectomy. ${ }^{16,19,20}$

Although it is well known that many factors, such as hepatocyte growth factor, epidermal growth factor, and transforming growth factor alpha, trigger hepatocyte proliferation after partial hepatectomy, it is not well known which factor (or

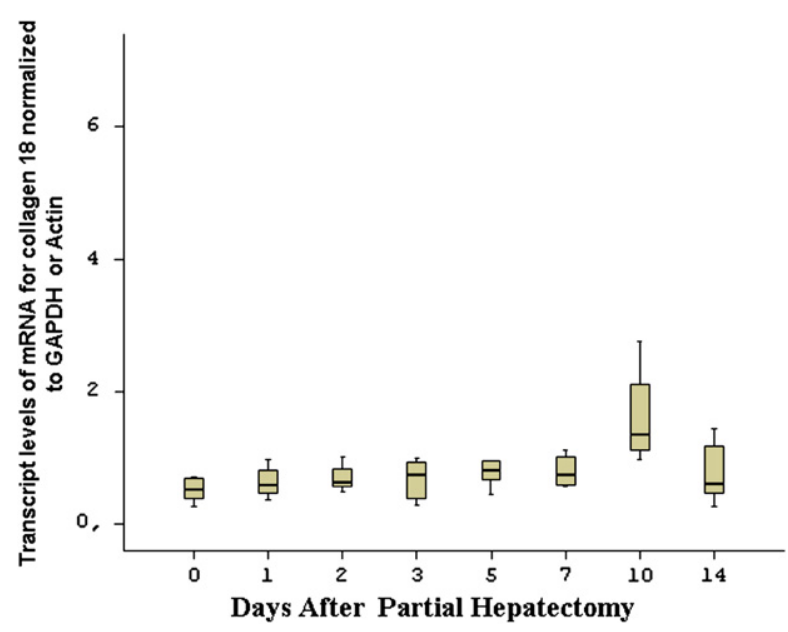

Fig 6. Transcript levels of mRNA for collagen 18 normalized to GAPDH or actin reached peak value on day 10 and returned to basal value on day 14 .

factors) eventually stops the liver regeneration after the liver reaches its optimal mass. Some studies have focused on TGF- $\beta 1$, a known inhibitor of proliferation in hepatocyte cultures ${ }^{2}$; however, hepatocyte proliferation stops between 48 and 72 hr, a time when they are still resistant to TGF- $\beta 1$. Therefore, TGF- $\beta 1$ is not sufficient to act as the terminating signal for liver regeneration. ${ }^{2}$ 


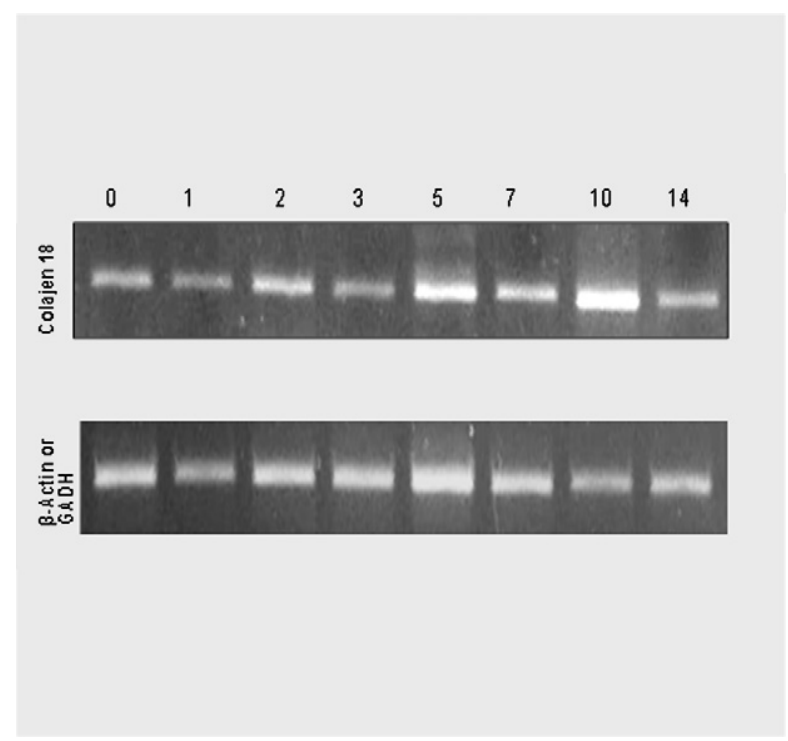

Fig 7. A sample from ultraviolet images obtained by RT-PCR of each animal was compared with corresponding $\beta$-actin or GAPDH.

Greene et al demonstrated that angiogenic agents modulate the liver regeneration of hepatic mass primarily by affecting endothelial cell proliferation or apoptosis. ${ }^{10}$ They hypothesized that endothelial cells may be the physiologic brake that stops liver regeneration based on the finding that endothelial apoptosis increased on the same day that the liver reached its pre-operative volume. ${ }^{10}$ From these findings, they suggested that endothelial cells were a key mediator of regenerating adult tissue mass in a partial hepatectomy model. ${ }^{10} \mathrm{Al}-$ though they evaluated the effect of exogenously applied endothelial cell specific agents on hepatic regeneration, they did not study endogenous agents.

In our study, we demonstrated that expression of mRNA for collagen 18 was present during liver regeneration after partial hepatectomy, indicative of antiangiogenic activity of hepatocytes. This is the first study to demonstrate antiangiogenic activity during normal liver regeneration in the remnant liver after partial hepatectomy in rats. We used C18 as a marker of antiangiogenic activity, because it is the precursor molecule of endostatin $(22 \mathrm{kDa})$, which is known to be an endogenous antiangiogenic agent. ${ }^{21,22} \mathrm{Oh}$ et al isolated a collagenous polypeptide and designated it as C18. ${ }^{14}$ In addition, they showed that C18 was present in multiple organs with the greatest level in the liver, lungs, and kidney. ${ }^{14}$ Later, the liver was shown to be the main site of C18 synthesis, and hepatocytes in the normal liver and satellite cells in the cirrhotic liver were reported to be the important sources of this type of collagen. ${ }^{13} \mathrm{C} 18$ expression is increased in some conditions such as hepatocellular carcinoma and liver fibrosis. As a result, it has been postulated that the precursor of endostatin might be expressed differently according to the differentiated and/or transformed state of hepatocytes. ${ }^{13,23,24}$ According to the findings of the present study, we suggest that there is baseline synthesis of C18 in hepatocyes ( 0.5 on day 0$)$, but that its expression is increased during liver regeneration.

Sasaki et al demonstrated the broad occurrence of endostatin-like proteins in tissues and serum with size ranges of $22 \mathrm{kDa}$ to $38 \mathrm{kDa}$. In fact, they showed that the noncollagenous domain (NC1) of C18 had 11 different positions in the hinge region sensitive to proteolysis. This structure may indicate that several different proteolytic pathways may exist for the generation of endostatin in tissues. ${ }^{25}$ Immunohistochemical studies have shown that molecules derived from C18 in tissues resemble $\mathrm{NC1}$, whereas in circulation they are more similar to endostatin. ${ }^{26}$ Also, it has been demonstrated that NC1 is released from parental collagen in many tissues, and that a second proteolytic step may be required to release soluble endostatin which could act in a paracrine or endocrine fashion. ${ }^{25}$

In another study, serum endostatin levels were greater on day 14 compared to day 1 after $40 \%$ and $70 \%$ hepatectomy in normal mice ${ }^{5}$; angiogenesis disappeared, however, on day 7. This high level of endostatin after cessation of angiogenesis was ascribed to the prolonged effect of endostatin. ${ }^{5}$ In contrast, we found that C18 mRNA expression in hepatocytes peaked on day 10, but returned to basal values on day 14 . This difference between the changes in serum endostatin levels and tissue C18 mRNA expression may be explained by the fact that C18 shows its antiangiogenic effect during liver regeneration in a paracrine fashion, but endostatin, of which C18 is the precursor molecule, may continue to be present in circulation for its endocrine function. Further studies are needed to determine tissue expression of C18 protein and serum endostatin levels during liver regeneration.

In conclusion, we demonstrated that C18 mRNA expression is present in the normal liver during the regenerative process. The stimulus that causes the cessation of the regeneration process may come from hepatocytes and collagen 18 produced by hepatocytes may modulate this event by inhibiting angiogenesis. The production of C18 protein and its functional activity during normal 
liver regeneration need to be demonstrated in further studies.

\section{REFERENCES}

1. Black D, Lyman S, Heider TR, Behrns KE. Molecular and cellular features of hepatic regeneration. J Surg Res 2004; 117:306-15.

2. Michalopoulos GK, DeFrances MC. Liver regeneration. Science 1997;276:60-6.

3. Kay MA, Fausto N. Liver regeneration: prospects for therapy based on new technologies. Mol Med Today 1997;3:108-15.

4. Kam I, Lynch S, Svanas G, Todo S, Polimeno L, Francavilla A, Penkrot RJ, et al. Evidence that host size determines liver size: studies in dogs receiving orthotopic liver transplants. Hepatology 1987;7:362-6.

5. Colakoglu T, Keskek M, Colakoglu S, Can B, Sayek I. Serum endostatin levels and regenerative capacities of normal and cirrhotic livers following partial hepatectomy in mice: the response to different resection sizes. J Surg Res 2007;143:337-43.

6. Abbasoglu O, Berker M, Ayhan A, Palaoglu S, Sayek I. The effect of the pineal gland on liver regeneration in rats. J Hepatol 1995;23:578-81.

7. Kahn D, Hickman R, Terblanche J, von Sommoggy S. Partial hepatectomy and liver regeneration in pigs-the response to different resection sizes. J Surg Res 1988;45:176-80.

8. Zieve L, Anderson WR, Lindblad S. Course of hepatic regeneration after $80 \%$ to $90 \%$ resection of normal rat liver. Comparison with two-lobe and one-lobe hepatectomy. J Lab Clin Med 1985;105:331-6.

9. Liekens S, De Clercq E, Neyts J. Angiogenesis: regulators and clinical applications. Biochem Pharmacol 2001;61:253-70.

10. Greene AK, Wiener S, Puder M, Yoshida A, Shi B, PerezAtayde AR, et al. Endothelial-directed hepatic regeneration after partial hepatectomy. Ann Surg 2003;237:530-5.

11. Ross MA, Sander CM, Kleeb TB, Watkins SC, Stolz DB. Spatiotemporal expression of angiogenesis growth factor receptors during the revascularization of regenerating rat liver. Hepatology 2001;34:1135-48.

12. O'Reilly MS, Boehm T, Shing Y, Fukai N, Vasios G, Lane WS, et al. Endostatin: an endogenous inhibitor of angiogenesis and tumor growth. Cell 1997;88:277-85.

13. Lietard J, Theret N, Rehn M, Musso O, Dargere D, Pihlajaniemi $T$, et al. The promoter of the long variant of collagen XVIII, the precursor of endostatin, contains liver-specific regulatory elements. Hepatology 2000;32:1377-85.

14. Oh SP, Kamagata Y, Muragaki Y, Timmons S, Ooshima A, Olsen BR. Isolation and sequencing of cDNAs for proteins with multiple domains of Gly-Xaa-Yaa repeats identify a distinct family of collagenous proteins. Proc Natl Acad Sci U S A 1994;91:4229-33.

15. Reynaert H, Chavez M, Geerts A. Vascular endothelial growth factor and liver regeneration. J Hepatol 2001;34:759-61.

16. Shimizu H, Miyazaki M, Wakabayashi Y, Mitsuhashi N, Kato A, Ito $\mathrm{H}$, et al. Vascular endothelial growth factor secreted by replicating hepatocytes induces sinusoidal endothelial cell proliferation during regeneration after partial hepatectomy in rats. J Hepatol 2001;34:683-9.

17. Drixler TA, Vogten MJ, Ritchie ED, van Vroonhoven TJ, Gebbink MF, Voest EE, et al. Liver regeneration is an angiogenesis-associated phenomenon. Ann Surg 2002; 236:703-11.

18. Higgins GM, Anderson RM. Experimental pathology of the liver: restoration of the liver of white rat following partial surgical removal. Arch Patol 1931;12:186-202.

19. Sato T, El-Assal ON, Ono T, Yamanoi A, Dhar DK, Nagasue N. Sinusoidal endothelial cell proliferation and expression of angiopoietin/Tie family in regenerating rat liver. J Hepatol 2001;34:690-8.

20. Taniguchi E, Sakisaka S, Matsuo K, Tanikawa K, Sata M. Expression and role of vascular endothelial growth factor in liver regeneration after partial hepatectomy in rats. J Histochem Cytochem 2001;49:121-30.

21. Saarela J, Ylikarppa R, Rehn M, Purmonen S, Pihlajaniemi T. Complete primary structure of two variant forms of human type XVIII collagen and tissue-specific differences in the expression of the corresponding transcripts. Matrix Biol 1998;16:319-28.

22. Saarela J, Rehn M, Oikarinen A, Autio-Harmainen H, Pihlajaniemi T. The short and long forms of type XVIII collagen show clear tissue specificities in their expression and location in basement membrane zones in humans. Am J Pathol 1998;153:611-26.

23. O'Reilly MS, Holmgren L, Chen C, Folkman J. Angiostatin induces and sustains dormancy of human primary tumors in mice. Nat Med 1996;2:689-92.

24. Cavallaro U, Christofori G. Molecular mechanisms of tumor angiogenesis and tumor progression. J Neurooncol 2000; 50:63-70.

25. Sasaki T, Fukai N, Mann K, Gohring W, Olsen BR, Timpl R. Structure, function and tissue forms of the C-terminal globular domain of collagen XVIII containing the angiogenesis inhibitor endostatin. Embo J 1998;17:4249-56.

26. Sasaki T, Larsson H, Tisi D, Claesson-Welsh L, Hohenester E, Timpl R. Endostatins derived from collagens XV and XVIII differ in structural and binding properties, tissue distribution and anti-angiogenic activity. J Mol Biol 2000;301: 1179-90. 